\title{
Effect of intravitreal hyaluronidase on the clearance of tritiated water from the vitreous to the choroid
}

\author{
WALLACE S FOULDS,' DONALD ALLAN, ${ }^{2}$ HARRY MOSELEY, \\ AND PETER M KYLE' \\ From the 'Tennent Institute of Ophthalmology, University of Glasgow, and the ${ }^{2}$ West of Scotland Health \\ Boards, Department of Clinical Physics and Bio-Engineering, Glasgow
}

SUMMARY The rate of transfer of intravitreally injected tritiated water from the mid vitreous to the choroid is significantly increased after depolymerisation of vitreous hyaluronic acid by injected hyaluronidase. The significance of this finding is discussed in relation to such conditions as retinal detachment and reattachment.

We have previously described a method for studying the transfer of intravitreally injected tracers from the vitreous to the choroid. The major proportion of intravitreally injected tritiated water is rapidly cleared to the choroid across the cortical vitreous, retina, and retinal pigment epithelium (RPE).' This movement of water from the vitreous to the choroid may be of importance in the maintenance of retinal apposition..$^{23}$ We were interested to see whether modification of the physical characteristics of the cortical vitreous gel by depolymerisation of its contained hyaluronic acid would alter the rate of transfer of tritiated water from the mid vitreous to the choroid.

Hyaluronic acid in the vitreous is an important structural component, ${ }^{+}$and, although this highly polymerised hydrophilic molecule is likely to interfere with bulk movement of water, it has been suggested that diffusional water movement would not be so influenced (Balazs E A, personal communication). In the present series of experiments we have measured the clearance of intravitreally injected ${ }^{3} \mathrm{H}_{2} \mathrm{O}$ in rabbit eyes in which the vitreous hyaluronic acid had been deploymerised by previously injected hyaluronidase and have compared the results with earlier measurements on normal rabbit eyes.'

\section{Materials and methods}

The experiments were performed on a group of six adult male Dutch rabbits weighing between 1.6 and

Correspondence to Professor W S Foulds, Tennent Institute of Ophthalmology, Western Infirmary, Glasgow G11 6NT.
$2.4 \mathrm{~kg}$ and compared with control experiments carried out on a group of nine animals of similar strain, age, and weight. The animals were anaesthetised with intravenous ethyl carbamate (Urethane) in a dose of $1.9 \mathrm{mg} / \mathrm{kg}$ body weight. Subsequently 750 international units of bovine testicular hyaluronidase (Hyalase; Fisons Ltd, Pharamaceutical Division) in $0.1 \mathrm{ml}$ water was injected via the pars plana ciliaris into the middle of the vitreous cavity of one eye with a microsyringe and a 26 gauge needle. The injecting needle was kept in situ during the rest of the experiment.

Approximately 30 minutes after the injection of hyaluronidase $25 \mu \mathrm{Ci}$ of ${ }^{3} \mathrm{H}_{2} \mathrm{O}$ in $25 \mu$ l of water was. injected into the mid vitreous through a separate injection site in the pars plana ciliaris, and this needle too was kept in situ while clearance of the injected ${ }^{3} \mathrm{H}_{2} \mathrm{O}$ to the choroid was measured by the technique previously described.' In summary, the upper nasal vortex vein was identified, cleared of surrounding tissue, and divided. Blood was collected from its cut end into long form Pasteur pipettes under the influence of capillarity and gravity, the pipettes being changed at two-minute intervals or more quickly if the blood flow was great enough to justify it. Blood samples were weighed, decolourised, and mixed with scintillation fluid (NE 260, Nuclear Enterprises Ltd), and the activity of ${ }^{3} \mathrm{H}$ in the blood samples was assayed by liquid scintillation counting. At the end of the experiment the animal was killed and the eye enucleated. The number of vortex veins was counted, and the state of the vitreous was determined by examination of the bisected globe with an operating microscope. 
In control experiments the experimental method was similar to that used in the hyaluronidase experiments, except that the injection of intravitreal hyaluronidase was omitted. The results of control experiments in normal eyes have been previously reported.' In relation to the state of the vitreous as examined under the operating miscroscope, the fellow eye in animals in which intravitreal hyaluronidase had been injected in one eye was used as a control.

\section{Results}

As had previously been found in control experiments, ${ }^{1}$ all eyes had four vortex veins. There was, however, a marked difference in the state of the vitreous in eyes receiving hyaluronidase as compared with normal rabbit eyes, the vitreous in the former being liquid and rapidly running out of the bisected globe, unlike the latter in which the vitreous retained its firm gel-like characteristics.

In our initial study of normal rabbits the parameter used to characterise the rate of appearance of the ${ }^{3} \mathrm{H}_{2} \mathrm{O}$ in choroidal blood was the mean transit time, the average time taken for a molecule of ${ }^{3} \mathrm{H}_{2} \mathrm{O}$ to be cleared from the eye. However, the value of this quantity depends on the duration of the experiment, and the use of this parameter is inappropriate where experiments of differing lengths must be compared. In the present study control experiments lasted 80 minutes and those on rabbits injected with hyaluronidase only 60 minutes.

The difficulty in making comparisons between these two groups of experiments was resolved by fitting an exponential decay to the decrease in ${ }^{3} \mathrm{H}$ concentration in the blood samples after the maximum value had been reached several minutes after the start of the experiment. Examples of fitted decays

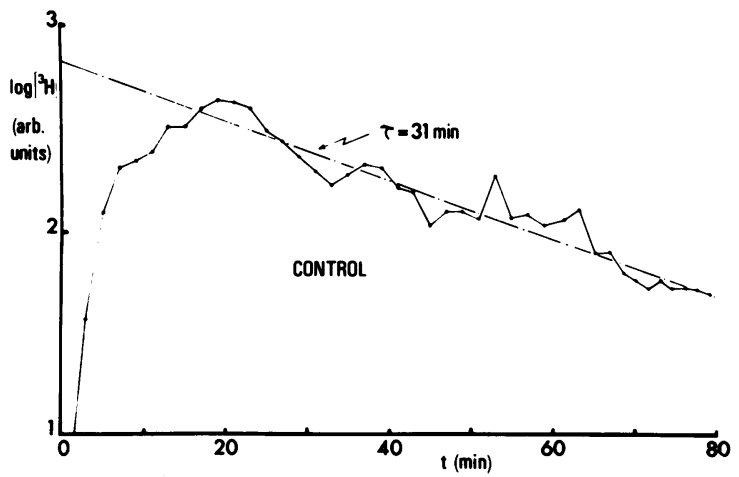

Fig. 1 Example of exponential decay fitted to rate of decline of ${ }^{3} \mathrm{H}$ concentration in vortex vein blood samples after intravitreal injection of ${ }^{3} \mathrm{H}_{2} \mathrm{O}$ in a normal rabbit eye.

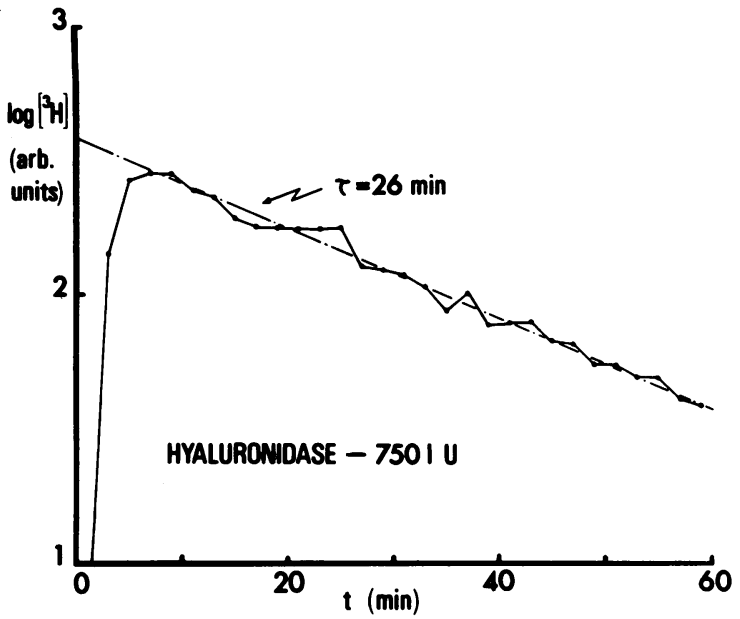

Fig. 2 Application of an exponential decay to the decline in ${ }^{3} \mathrm{H}$ concentration in blood samples from a vortex vein after an intravitreal injection of ${ }^{3} \mathrm{H}_{2} \mathrm{O}$ in an eye in which the vitreous hyaluronic acid had been depolymerised by the injection of hyaluronidase.

are shown in Figs. 1 and 2 by the dot-dash lines and it is evident that these do give a good description of the variation of the data over much of the experimental time. We therefore took the time constant, $\tau$, for the fitted decay as the index of rapidity of clearance of ${ }^{3} \mathrm{H}$. $\tau$ has no intrinsic dependence on the length of experiment; smaller values of $\tau$ correspond to a more rapid removal of tritiated water.

The values of $\tau$ obtained from the experiments on the rabbits treated with hyaluronidase are detailed in Table 1 together with values obtained from reanalysing the control experiments described previously.

Table 1 Rate of movement of ${ }^{3} \mathrm{H}_{2} \mathrm{O}$ from vitreous to choroid in normal rabbit eyes and in eyes injected with intravitreal hyaluronidase. The rate of clearance of ${ }^{3} \mathrm{H}_{2} \mathrm{O}$ from vitreous to choroid is increased in hyaluronidase treated eyes

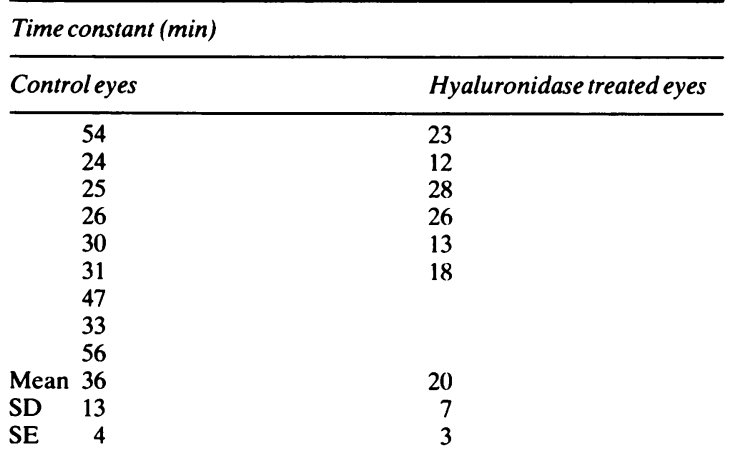

$0.01<\mathrm{P}<0.02$ (Mann-Whitney test). 
Table 2 Total recovery of ${ }^{3} \mathrm{H}_{2} \mathrm{O}$ from one vortex vein after intravitreal injection of ${ }^{3} \mathrm{H}_{2} \mathrm{O}$ in normal eyes and in eyes receiving an intravitreal injection of hyaluronidase

\begin{tabular}{|c|c|}
\hline Control eyes & Hyaluronidase treated eyes \\
\hline 18 & 30 \\
\hline 38 & 54 \\
\hline 23 & 14 \\
\hline 35 & 14 \\
\hline 27 & 13 \\
\hline 24 & 12 \\
\hline 26 & \\
\hline 24 & \\
\hline 13 & \\
\hline Mean 25 & 23 \\
\hline SD 8 & 17 \\
\hline $\mathrm{SE}$ & 7 \\
\hline
\end{tabular}

$0 \cdot 2<\mathrm{P}<0 \cdot 5$ (Mann-Whitney test).

The mean time constant for the hyaluronidase series was $20 \pm 3$ minutes (mean \pm standard error) compared with $36 \pm 4$ minutes for the control group. The difference between the two sets of time constants was tested by the non-parametric Mann-Whitney test and found to be significant at the $5 \%$ level $(0 \cdot 01<\mathrm{p}<0 \cdot 02$; two-tailed $)$.

The total recoveries of ${ }^{3} \mathrm{H}_{2} \mathrm{O}$ are set out in Table 2 with the control data for comparison. A MannWhitney test showed no significant difference between the two groups of data, though the trend was for lower recoveries in the hyaluronidase series perhaps because of the shorter durations of experiments in this group.

\section{Discussion}

Although the results of the experiments on water movement from the vitreous to the choroid in normal control animals fitted the pattern expected for diffusional movement of water from vitreous to the choroid, we have not excluded the possibility of a pressure-dependent component of flow or indeed a movement dependent upon active transport in the pigment epithelium or resulting from the colloid osmotic pressure difference between the vitreous and the protein-rich extravascular spaces of the choroid.

The control animals used in these experiments were of the same strain, weight, and age as the animals receiving intravitreal hyaluronidase, and the experiment itself was similar in both groups of animals with the exception of the introduction of hyaluronidase into the eyes of the experimental group. In some respects it might have been better to have used the fellow eye of the same animal as a control, but this was precluded by the impossibility of achieving adequate access to vortex veins in each eye simultaneously. The question of leakage round the injection sites of either the tritiated water or the hyaluronidase had also to be considered. Experiments in which fluorescein was mixed with the injectate showed no evidence of leakage round the injection sites when the technique described was used, nor were significant differences found between the recovered amounts of injectate in control and experimental eyes (Table 2), which would have been expected had there been significant loss of injectate round the injection site in eyes receiving intravitreal hyaluronidase.

From the data presented here it does appear that vitreous hyaluronic acid can influence the diffusional movement of water in the vitreous. In the normal eye the polymerised hyaluronic acid is evidently a rate limiting factor, possibly one of several, in the processes of water transport from mid vitreous to choroid, since the rate of water transfer has been shown to be faster in eyes treated with hyaluronidase, and it is known that hyaluronidase depolymerises vitreous hyaluronic acid. ${ }^{56}$

It has long been accepted that the vitreous structure could act as a barrier to the passage through it of large protein molecules, cells, and the like, and indeed it has been shown that hyaluronic acid in various concentrations systematically reduces the diffusion of chemotactic peptides in solution ${ }^{7}$ and also the movement of macrophages. ${ }^{\circ}$ The question of the influence of hyaluronic acid on water movement has been less certain. Ogston and Sherman" showed that solutions of hyaluronic acid stabilised on micropore filters could slow down water flow, but Balazs (personal communication) has refuted the suggestion that hyaluronic acid in the vitreous would influence diffusional movement of water. On clinical grounds it has been suggested that intact vitreous gel may be capable of blocking open retinal holes, in some cases preventing retinal detachment." It has also been postulated that vitreous tamponade of retinal holes in volume-reducing operations for retinal detachment may be important in relation to surgical cure of such detachments. "I In particular it has been suggested that vitreous tamponade of a retinal hole might explain the flattening of the retina which commonly occurs after episcleral plombage where the retinal hole is not in contact with the indentation in the immediate postoperative period. ${ }^{\prime \prime \prime}$

The present study lends support to the view that intact cortical vitreous gel can limit the movement of water molecules, confirming that vitreous tamponade of retinal holes is a physical possibility.

We thank the British Medical Association (John Clarke award) for financial support and Mrs J Murray for typing the manuscript. 


\section{References}

1 Moseley H, Foulds WS, Allan D, Kyle PM. Routes of clearance of radioactive water from the rabbit vitreous. $\mathrm{Br} J$ Ophthalmol 1984; 68: 145-51.

2 Foulds WS. Aetiology of retinal detachment. Trans Ophthalmol Soc UK 1975; 95: 118-27.

3 Frambach DA, Marmor MF. The rate and route of fluid absorption from the subretinal space of the rabbit. Invest Ophthalmol Visual Sci 1982; 22: 292-302.

4 Balazs EA. Physiology of the vitreous body. In: Schepens CL, ed. The importance of the vitreous body in retina surgery. St Louis, Mosby: 1960: 29-48.

5 Boruchoff SA, Woodin AM. Viscosity and composition of solutions derived from rabbit vitreous humour. $\mathrm{BrJ}$ Ophthalmol 1956; 40: 113-8.

6 Rowen JW, Brunish R, Bishop FW. Form and dimensions of isolated hyaluronic acid. Biochim Biophys Acta 1980; 19: 480-9.

7 Forrester JV, Wilkinson PC. Inhibition of leucocyte locomotion by hyaluronic acid. J Cell Sci $1981 ;$ 48: 313-31.

8 Forrester JV, Balazs EA. Inhibition of phagocytosis by high molecular weight hyaluronate. Immunology 1980; 40: 435-46.

9 Ogston AG, Sherman TF. Effects of hyaluronic acid upon diffusion of solutes and flow of solvent. J Physiol (Lond) 1961; 156: 67-74.

10 Foulds WS. The vitreous in retinal detachment. Trans Ophthalmol Soc UK 1975; 95: 412-6. 